\title{
Cytogenetic Studies in Myeloproliferative Disorders ${ }^{1,2}$
}

\author{
Digamber S. Borgaonkar \\ Division of Medical Genetics, Department of Medicine, \\ The Johns Hopkins University School of Medicine, \\ Baltimore, Maryland 21205, U.S.A.
}

Received September 2, 1970

On clinical and hematological grounds the myeloproliferative disorders have been subdivided into at least 6 entities: polycythemia vera, chronic myeloid leukemia, myelofibrosis with myeloid metaplasia, thrombocythemia, acute myeloid leukemia, and di Guglielmo's syndrome (Dameshek 1951, Dameshek and Gunz 1964). However, these disorders have overlapping clinical, pathological, and hematological features; and are often difficult to differentiate. In general, they result from the abnormal proliferation, either in the bone marrow or potential extra-medullary sites, of one or more marrow elements. Since the discovery of the minute (later termed "Philadelphia" or $\mathrm{Ph}^{1 ")}$ ) chromosome in chronic myeloid leukemia by Nowell and Hungerford (1960), numerous cytogenetic studies have been reported in myeloproliferative disorders. Recent reports of $\mathrm{Ph}^{+}$(positive) and $\mathrm{Ph}^{-}$(negative) cases of chronic myeloid leukemia (Krauss, Sokal, and Sandberg 1964, Tijio et al. 1966) and the occurrence of the $\mathrm{Ph}^{1}$ chromosome in myelofibrosis (Bowen and Lee 1963), myeloid metaplasia (Forrester and Louro 1966), and eosinophilic leukemia (Gruenwald et al. 1965) have raised the question of the specificity of the $\mathrm{Ph}^{1}$ chromosome in myeloproliferative disorders (Goh and Swisher 1964, Heath and Moloney 1965). Trisomy of a chromosome in the C (X+6-12) group has been reported in patients with myeloproliferative disorders (Nowell and Hungerford 1962, Winkelstein, Sparkes, and Craddock 1966), myelofibrosis with myeloid metaplasia and atypical panmyelosis (Kiossoglou, Mitus, and Dameshek 1966), bone marrow dysplasia (Lawler, Kay, and Birbeck 1966), myeloid metaplasia with possible leukemia (Sandberg et al. 1964), and preleukemia (Rowley, Blaisdell, and Jacobson 1966). On the other hand, monosomy of a C group chromosome has been reported in myelofibrosis with myeloid metaplasia (Jackson and Higgins 1967).

The present study analyses cytogenetic findings in a group of patients with myeloproliferative disorders and evaluates the significance of various karyotypic changes. Of particular interest is the presence of C-group trisomy in a patient with a long history of hematological disorders culminating in myeloid metaplasia, and a 46 chromosome karyotype with a missing $\mathrm{D}$ group chromosome and an extra $\mathrm{C}$ group chromosome in a patient with chronic monocytic leukemia. The $\mathrm{Ph}^{1}$

1 Presented in part at the Third International Congress of Human Genetics, Chicago, September, 1966 (Borgaonkar 1966).

2 Part of the study was supported by grant USPHS GM 10189. 
chromosome was found in 6 of 9 patients clinically diagnosed as having chronic myeloid leukemia.

\section{Materials and methods}

Eighteen patients with chronic myeloid leukemia, myeloid metaplasia, or chronic monocytic leukemia were studied from September, 1964 to December, 1966. Chronic monocytic leukemia was included because, like other myeloproliferative disorders, it may present as a refractory anemia, or demonstrate abnormally appearing platelets before a specific diagnosis can be established. These patients were either previously diagnosed on hematological and clinical grounds or were in the process of diagnostic evaluation. Ten were males and eight were females (Table 1).

Cytogenetic studies were performed by one or both of the following methods: direct preparations were obtained from bone marrow following the technique of Tjio and Whang (1962), and/or whole blood cultures were set up with and without the mitotic stimulating agent phytohemagglutinin (PHA) by a modification of the technique of Lejeune (1965). Blood cultures without PHA were harvested after 48 hours (Nowell and Hungerford 1964, Lewis et al. 1965). At least three karyotypes were examined for each patient.

The number of patients in each category is given below. Diagnoses were made independently of the results of cytogenetic study.

$\begin{array}{lr}\text { Chronic myeloid leukemia } & 9 \\ \text { Myeloid metaplasia (MM) } & 7 \\ \text { Chronic monocytic leukemia } & \frac{2}{18} \\ \text { TOTAL } & \frac{18}{}\end{array}$

\section{Results}

\section{Chronic meyloid leukemia}

The $\mathrm{Ph}^{1}$ chromosome was detected in 6 of 9 cases of CML diagnosed on clinical and hematological grounds (Table 1) (Fig. 1). In this group all peripheral blood cultures without PHA demonstrated the $\mathrm{Ph}^{1}$ chromosome. The other 3 cases had normal karyotypes in blood cultures without PHA, although one patient (No. 1) showed breaks and fragments in 50 per cent of her cells. She had had X-ray therapy and chemotherapy (Myleran) prior to the chromosome study. This patient also showed 20 per cent endoreduplicated cells.

\section{Myeloid metaplasia}

Seven cases of MM were studied (Table 1). A 39 year old female patient (No. 13) consistently showed 47 chromosomes in short-term (48-hour) blood cultures without phytohemagglutinin (Fig. 2). The extra chromosome was believed to be an autosome in the $C(X+6-12)$ group. Buccal smear from both sides showed a single sex-chromatin body in 29 per cent of cells. Blood cultures stimulated with PHA showed a normal female 46/XX karyotype (Table 1). Family history was noncontributory. The patient, one of five sibs, was married, had one normal 

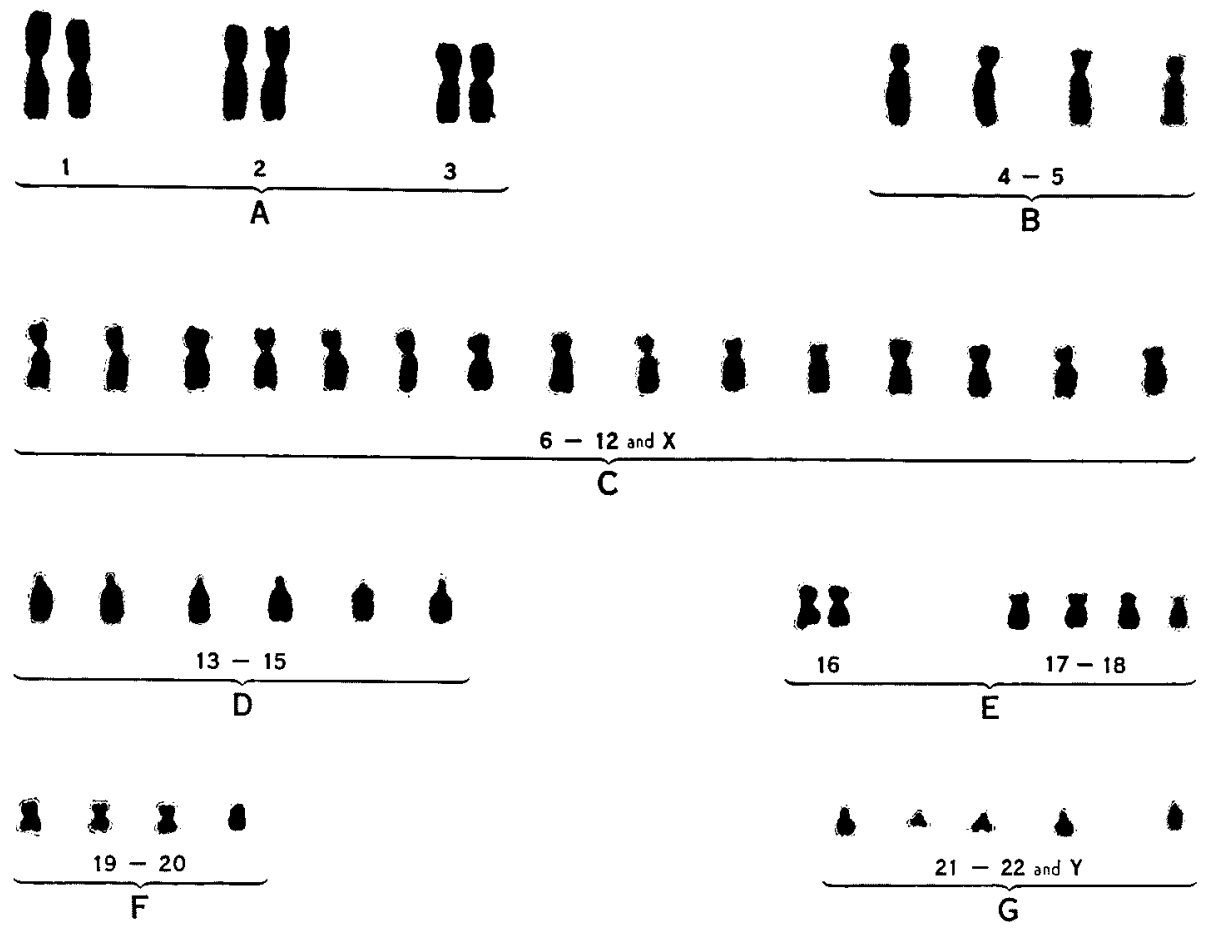

Fig.1. Karyotype of a cell from blood culture of patient No. 3 with CML showing the minute,
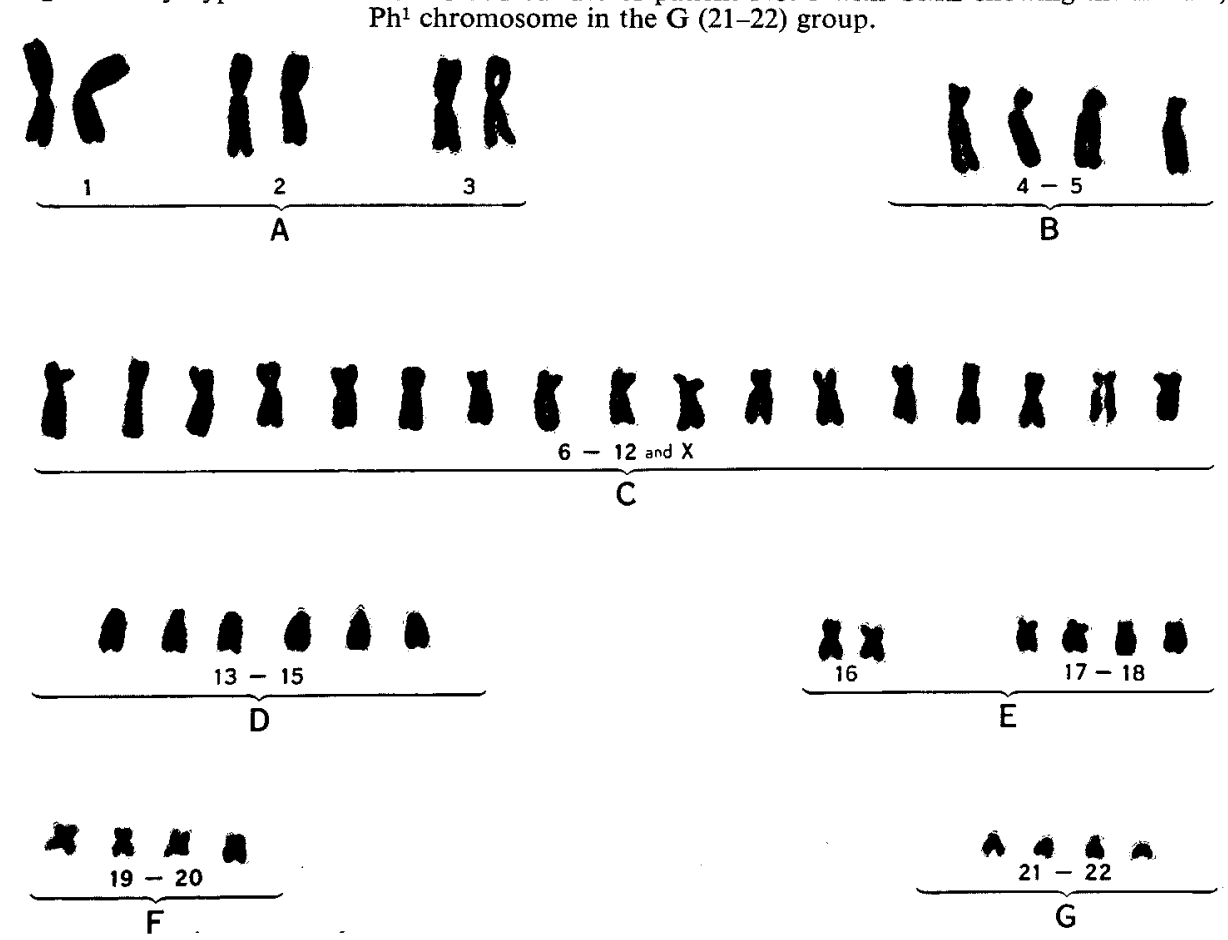

Fig. 2. Karyotype of a cell of patient No. 13(MM) showing trisomy of an autosome in the $\mathrm{C}(\mathrm{X}+$ 6-12) group. 


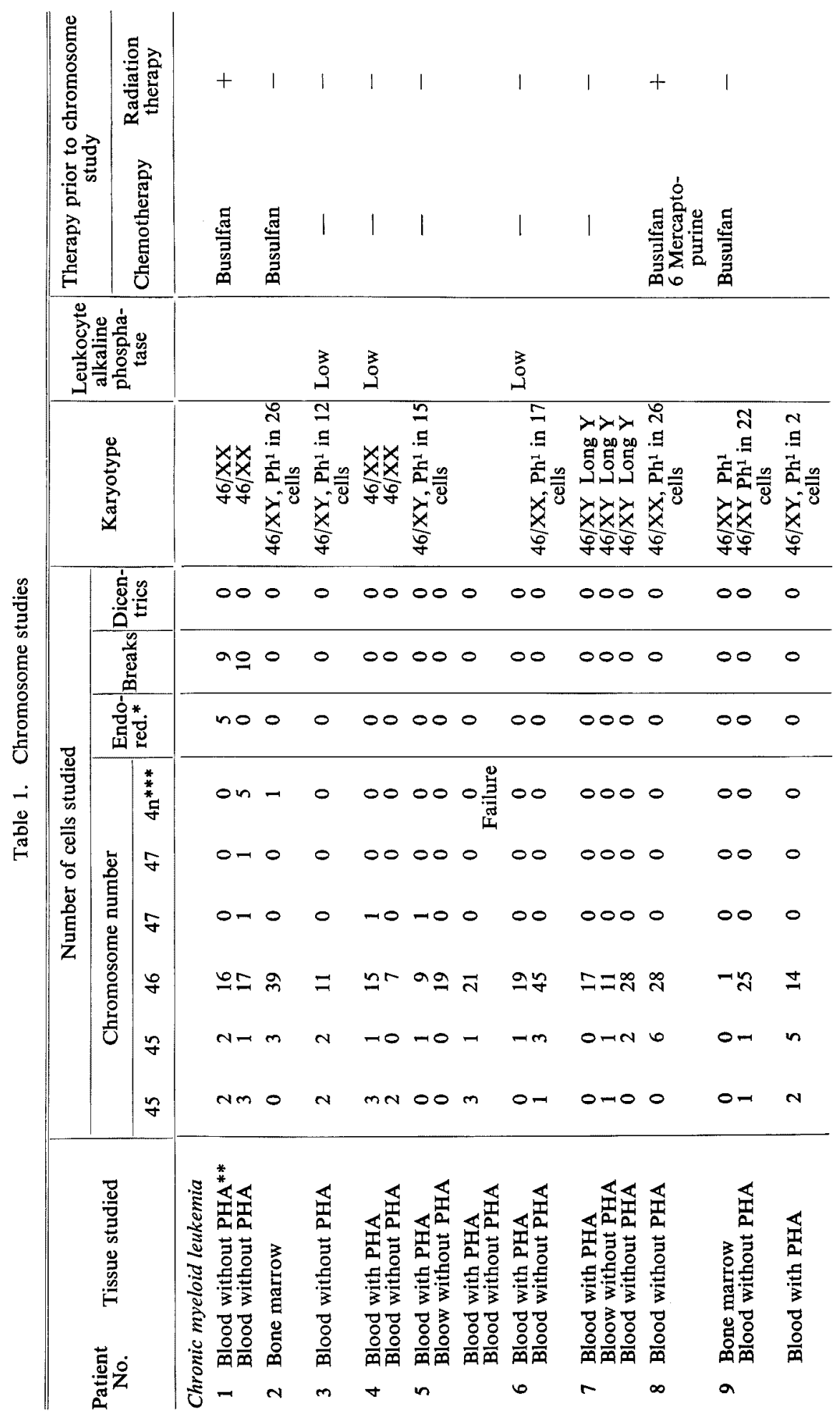




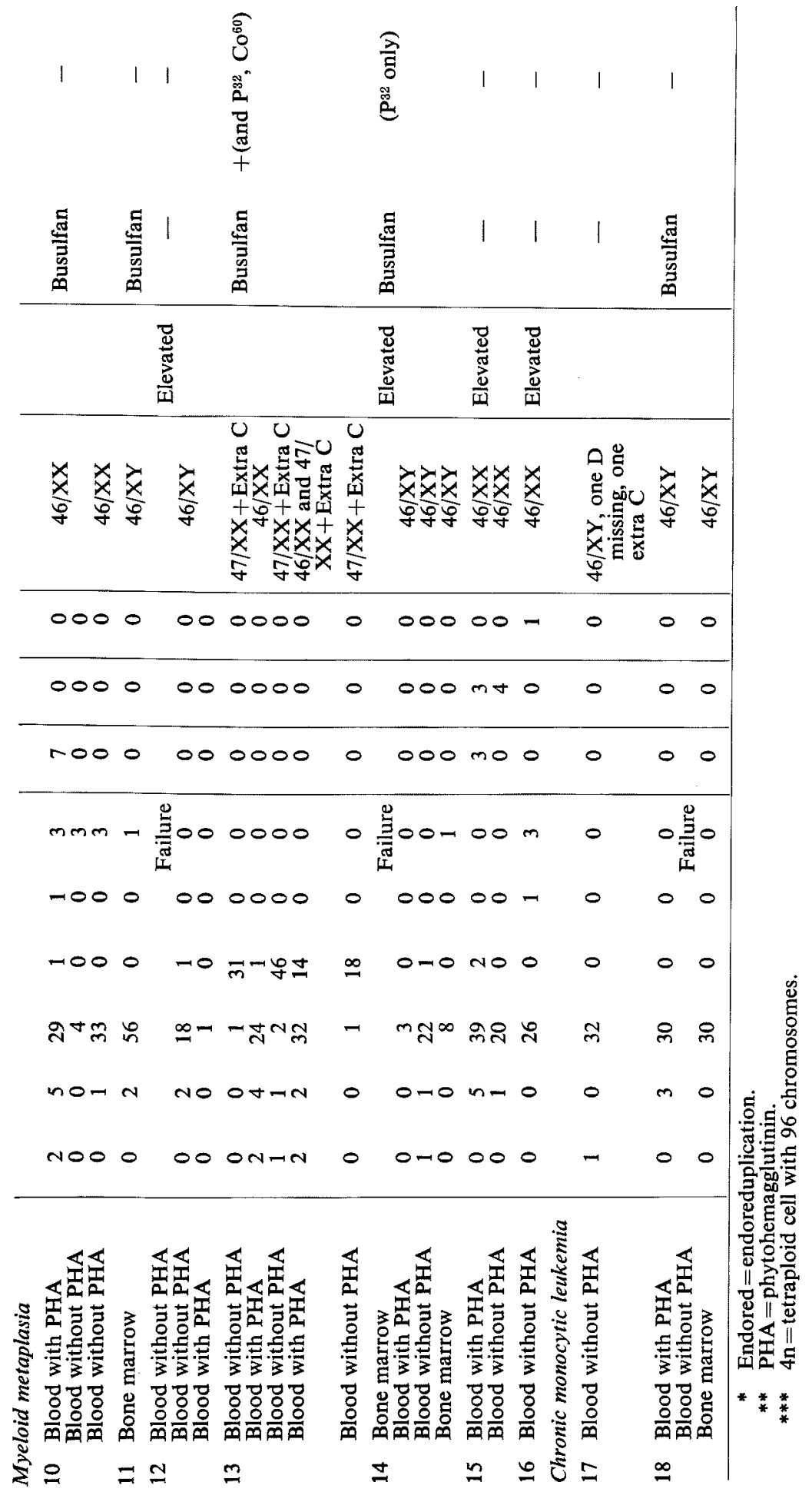


son, and had had four abortions. One patient (No. 15) showed breaks, fragments, and abnormal chromosomes.

\section{Chronic monocytic leukemia}

Two cases of chronic monocytic leukemia were studied (Table 1). One patient (No. 17) had 46 chromosomes but an analysis of the karyotypes showed that a D
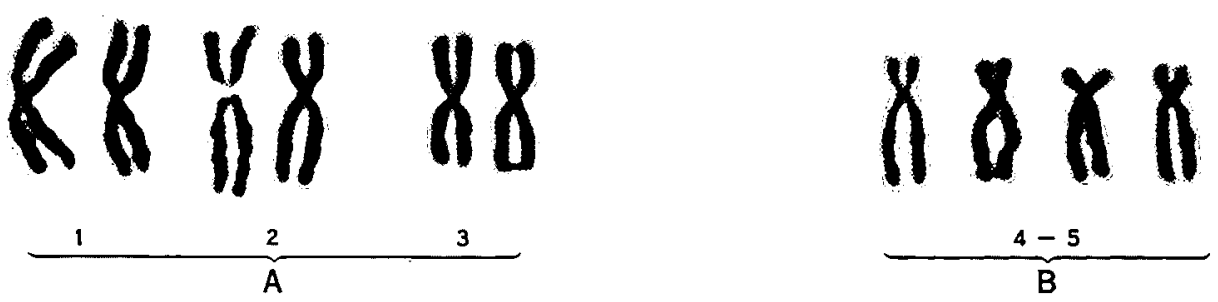

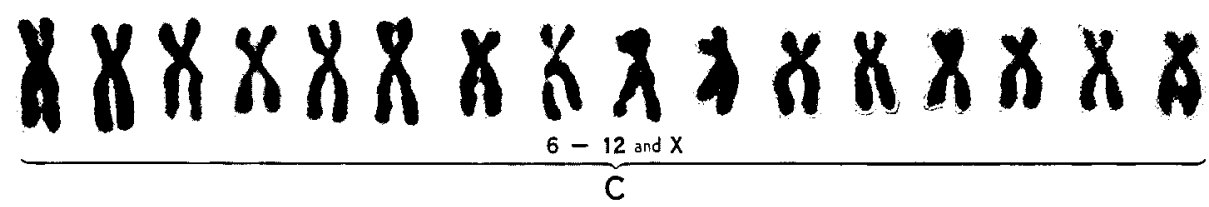
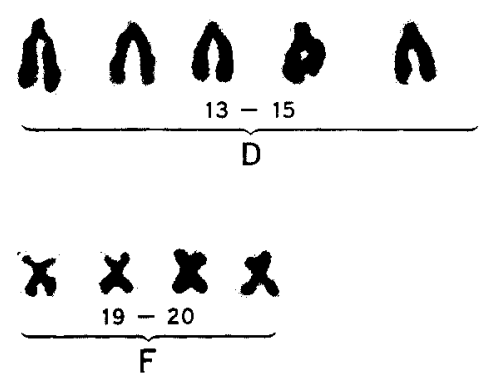
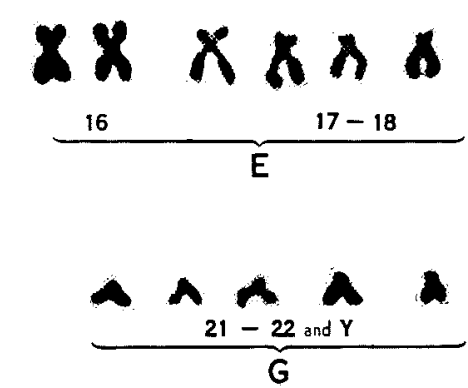

Fig. 3. Karyotype of a cell of patient No. 17 (chronic monocytic leukemia) with 46 chromosomes but with loss of a $\mathrm{D}$ group chromosome and addition of a $\mathrm{C}$ group chromosome.

(13-15) group chromosome was missing and an extra chromosome was present in the $\mathrm{C}(\mathrm{X}+6-12)$ group (Fig. 3). This short-term (48-hour) culture was set up without PHA. The pateient was married and had three apparently normal children. He died 10 weeks after the chromosome studies were initiated, at which time the clinical picture was that of acute monocytic leukemia.

\section{Disscussion}

The myeloproliferative disorders constitute a heterogeneous group in which the common feature is an abnormal proliferation of one or more bone marrow elements. The frequently overlapping clinical picture, hematological findings, chromosomal abnormality (e.g., occurrence of $\mathrm{Ph}^{1}$ chromosome in conditions other than CML), and leukocyte alkaline phosphatase levels make differentiation difficult (Dameshek 1951, Dameshek and Gunz 1964). The entities may be dis- 
tinct, but the criteria with which to distinguish one from another are not yet recognized.

The Philadelphia $\left(\mathrm{Ph}^{1}\right)$ chromosome was detected in six of nine patients with CML. The $\mathrm{Ph}^{1}$ chromosome was satisfactorily demonstrated either in direct bone marrow preparations or in 48-hour whole blood cultures set up without PHA. In such cultures chromosomes from the myeloid series of white blood cells are examined (Nowell and Hungerford 1964). With PHA the normal lymphocytes are stimulated; hence it is difficult and sometimes impossible to obtain $\mathrm{Ph}^{1}$ positive cells in PHA stimulated cultures (Lewis et al. 1967) (Table 1).

In three cases of $\mathrm{CML}$ the $\mathrm{Ph}^{1}$ chromosome was not found in blood cultures without PHA. Since bone marrow examinations could not be carried out in these three patients one cannot be absolutely certain that they were $\mathrm{Ph}^{1}$ negative. However, it is very likely that the mitotic figures observed represented immature cells which were dividing in vivo, i.e., leukemic cells (Nowell and Hungerford 1964). The finding of $\mathrm{Ph}^{1}$ negative CML confirms the previous report of similar cases by Tjio et al. (1966). In two of these cases (Nos. 4 and 7), the unequivocal diagnosis of CML was made at post-mortem examination. Previous bone marrow examination and differential blood counts had suggested the possibility that the patients had myelofibrosis with myeloid metaplasia. The leucocyte alkaline phosphatase (LAP) level in one of these patients (No. 4), however, was low, supporting the diagnosis of CML. These two cases illustrate how difficult it can be to distinguish accurately the different myeloproliferative disorders, particularly since the character of the disease may change during the course of the illness (Krasilnikoff 1967, Carbone et al. 1963).

The finding of a $\mathrm{C}(\mathrm{X}+6-12)$ group chromosome trisomy in one case of myeloid metaplasia (No. 13) is of interest. This patient had a 12-year history of polycythemia vera and had received chemotherapy (Busulfan) and radiation therapy $\left(\mathrm{P}^{32}, \mathrm{CO}^{60}\right.$, and $\mathrm{X}$-ray) prior to chromosome study. Her karyotype was normal when blood cultures were stimulated with PHA. The 47-chromosome cell-line, however, was predominant in blood cultures without PHA. Kiossoglou et al. (1966) have reported C trisomy in patients with myelofibrosis and myeloid metaplasia and observed that this "may determine patterns of myelofibrosis with myeloid metaplasia or of atypical panmyelosis." C trisomy has also been observed in acute leukemia (Sandberg et al. 1962). Winkelstein, Sparkes and Craddock (1966) found $\mathrm{C}$ trisomy in bone marrow preparations from a patient with leukocytosis with myeloid immaturity, thrombocytopenia, ineffective extra-medullary erythropoieses, overt hemolysis and decreased leukocyte alkaline phosphatase (LAP) activity. Two other similar cases have been reported (Sandberg et al. 1962, Nowell and Hungerford 1962). Our patient showed a single sex chromatin body in buccal mucosa cells and was otherwise normal, and we have therefore concluded that the extra chromosome was not an $\mathrm{X}$ chromosome but rather an autosome, and an abnormality associated with the patient's hematologic disorder. This chromosomal abnormality appears to be a feature of more than one myeloproliferative disorder. It may be a cytogenetic abnormality comparable to the $\mathrm{Ph}^{1}$ chromosome which can no longer be considered pathognomonic of CML. Sandberg (1966), 
reviewing the role of chromosomes in leukemia and cancer, suggests, "In a way, the $\mathrm{Ph}^{1}$ abnormality would be similar to that of $\mathrm{C}$ group trisomy. ..." Alternatively, trisomy of the C (6-12) group may be a characteristic feature of a certain type of myeloproliferative disorder which has not yet been defined.

The finding of breaks, fragments, and abnormal chromosomes in two patients (Nos. 1 and 15) is not unexpected since it is well known that patients receiving either chemo- or radiation- therapy may show such abnormal cytological features (Bender and Gooch 1962). Cytological studies of patients after such treatment should be interpreted with caution, as chromosomal abnormalities found in such patients may not be characteristic of their disorder.

The abnormal karyotype found in a patient (No. 17) with chronic monocytic leukemia is worthy of comment. The patient had no obvious clinical abnormalities other than his hematological disease. The 46-chromosome cell line was consistent in all the cells analyzed. However, there was a missing D (13-15) chromosome and an extra chromosome in the $C(X+6-12)$ group. This could represent 1) trisomy of a $C$ group chromosome, 2) $D / G$ interchange with $G_{1}$ trisomy, or 3) interchange of a D group chromosome with any other chromosome. The second alternative is unlikely in the present case because the patient did not have Down's syndrome. The third alternative is also unlikely since he was the father of three apparently normal children in one of whom a normal karyotype was demonstrated. If the patient had an interchange chromosome, the chance of his transmitting an abnormal chromosome would have been 50 per cent. This karyotype is therefore best explained by postulating two simultaneous chromosomal aberrations during or after the onset of his present condition: loss of a D (13-15) group chromosome and addition of a $\mathrm{C}$ group chromosome.

\section{Summary}

Cytogenetic studies of bone marrow preparations and/or whole blood cultures were carried out in 18 patients with myeloproliferative disorders. The $\mathrm{Ph}^{1}$ chromosome was detected in six of nine patients with chronic myeloid leukemia. Chromosomal abnormalities included: group $\mathrm{C}$ trisomy in a patient with myeloid metaplasia, and a 46 chromosome karyotype but with a missing D group and an extra $\mathrm{C}$ group chromosome in a patient with chronic monocytic leukemia.

\section{Acknowledgments}

The author wishes to thank the following for allowing him to study their patients: Drs. C. L. Conley, J. R. Krevans, E. E. Morse, W. Schlott, and E. W. Smith; and thank Dr. C. L. Conley and Dr. V. A. McKusick for their interest and encouragement. He is indebted to Dr. Jack Levin of the Division of Hematology for the laboratory findings, interpretation, and helpful discussions. The technical assistance of Mrs. M. Arendar, Mrs. K. Johnson, Mrs. J. Lutz, Mrs. B. Lewis, Mrs. S. Holcomb, Miss H. Herr, and Mrs. E. Mules is appreciated. 


\section{References}

Bender, M. A. and Gooch, P. A. 1962. Persistent chromosome aberrations in irradiated human subjects. Radiation Research 16: 44-53.

Borgaonkar, D. S. 1966. Chromosome studies in myeloproliferative disorders. Abstracts of contributed papers to the Third Cong. Hum. Genet: 11.

Bowen, P. and Lee, C. S. N. 1963. $\mathrm{Ph}^{1}$ chromosome in the diagnosis of chronic myeloid leukemia: Report of a case with features simulating myelofibrosis. Bull. Johns Hopkins Hosp. 113: 1-12.

Carbone, P. P., Tjio, J. H., Whang, J., Block, J. B., Kremer, W. B. and Frei, E. A. 1963. The effect of treatment in patients with chronic myelogenous leukemia-hematologic and cytogenetic studies. Ann. Int. Med. 59: 622-628.

Dameshek, W. 1951. Some speculations on the myeloproliferative syndromes. Editorial, Blood 6: 372 .

- and Gunz, F. A. 1964. Leukemia, 2nd Ed. Grune and Stratton, New York, p. 356.

Forrester, R. H. and Louro, J. M. 1966. Philadelphia chromosome abnormality in agnogenic myeloid metaplasia. Ann. Intern. Med. 64: 622-627.

Goh, K. and Swisher, S. N. 1964. Specificity of the Philadelphia chromosome. Cytogenetic studies in cases of chronic myelocytic leukemia and myeloid metaplasia. Ann. Intern. Med. 61 : 609-624.

Gruenwald, H., Kiossoglou, K. A., Mitus, W. J. and Dameshek, W. 1965. Philadelphia chromosome in eosinophilic leukemia. Amer. J. Med. 39: 1003-1010.

Heath, C. W. and Moloney, W. C. 1965. The Philadelphia chromosome in an unusual case of myeloproliferative disease. Blood 26: 471.

Jackson, J. F. and Higgins, L. C. 1967. Group C monosomy in myelofibrosis with myeloid metaplasia. Arch. Intern. Med. 119: 403-406.

Kiossoglou, K. A., Mitus, W. J. and Dameshek, W. 1966. Cytogenetic studies in the chronic myeloproliferative syndrome. Blood 28: 241-252.

Krasilnikoff, P. A. 1967. Myelofibrosis and myeloid metaplasia. Acta Paediat. Scand. 76: $424-429$.

Krauss, S., Sokal, J. E. and Sandberg, A. A. 1964. Comparison of Philadelphia chromosomepositive and -negative patients with chronic myelocytic leukemia. Ann. Intern. Med. 61: $625-635$.

Lawler, S. D., Kay, H. E. M. and Birbeck, M. S. C. 1966. Marrow dysplasia with C trisomy and anomalies of the granulocyte nuclei. J. Clin. Path. 19: 214-219.

Lejeune, J. 1965. In R. Turpin and J. Lejeune, Les Chromosomes Humaines, Paris-GauthierVillars, p. 535.

Lewis, F. J. W., Andres, M. J., Faint, S. and MacTaggart, M. 1967. Direct and short-term culture techniques in the recognition of chromosomal abnormalities in acute leukemia and associated states. Proc. 10th Europ. Soc. Haemat., Strasbourg, 1965 Part II, 498-508.

Nowell, P. C. and Hungerford, D. A. 1960. A minute chromosome in human chronic granulocytic leukemia. Science 132: 1497.

- and Hungerford, D. A. 1962. Chromosome studies in human leukemia IV. Myeloproliferative syndrome and other atypical myeloid disorders. J. Nat. Cancer Inst. 29:911-931.

- and Hungerford, D. A. 1964. Chromosome changes in human leukemia and a tentative assessment of their significance. Ann. N.Y. Acad. Sci. 113: 654-662.

Rowley, J. D., Blaisdell, R. K. and Jacobson, L. O. 1966. Chromosome studies in preleukemia I. Aneuploidy of group $\mathrm{C}$ chromosomes in three patients. Blood 27: 782-799.

Sandberg, A. A., Ishihara, T., Crosswhite, L. H. and Hauschka, T. S. 1962. Comparison of chromosome constitution in chronic myelocytic leukemia and other myeloproliferative disorders. Blood 20: 293-423.

Sandberg, A. A., Ishihara, T., Kikuchi, Y. and Crosswhite, L.H. 1964. Chromosomal differences among the acute leukemias. Ann. N.Y. Acad. Sci. 113: 663-716. 
Sandberg, A. A. 1966. The chromosomes and causation of human cancer and leukemia. Cancer Res. 26: 2064-2081.

Tjio, J. H. and Whang, J. 1962. Chromosome preparations of bone marrow cells without prior in vitro culture or in vitro colchicine administration. Stain Tech. 37: 17-20.

-, Carbone, P. P., Whang, J. and Frei, E. 1964. The Philadelphia chromosome and chronic myelogenous leukemia. J. Nat. Cancer Inst. 36: 567-584.

Winkelstein, A., Sparkes, R. S. and Craddock, C. G. 1966. Trisomy of group C in a myeloproliferative disorder. Report of a case. Blood 27: 722-733. 